\title{
HI-rich dwarf galaxies in the Hydra I cluster
}

\section{Spectroscopic data}

\author{
P.-A. Duc ${ }^{1}$, V. Cayatte ${ }^{2}$, C. Balkowski ${ }^{2}$, T. X. Thuan ${ }^{3}$, P. Papaderos ${ }^{4}$, and W. van Driel ${ }^{5}$ \\ 1 CNRS URA 2052 and CEA, DSM, DAPNIA, Service d'astrophysique, 91191 Gif-sur-Yvette Cedex, France \\ 2 Observatoire de Paris, DAEC, UMR 8631, CNRS and Université Paris 7, 92195 Meudon Cedex, France \\ 3 Astronomy Department, University of Virginia, Charlottesville, VA 22903, USA \\ 4 Universitäts-Sternwarte, Geismarlandstr. 11, 37083 Göttingen, Germany \\ 5 Unité Scientifique Nançay, CNRS USR B704, Observatoire de Paris, 92195 Meudon Cedex, France
}

Received 8 December 2000 / Accepted 18 January 2001

\begin{abstract}
We present spectrophotometric data for a sample of $15 \mathrm{H} \mathrm{I-selected} \mathrm{dwarf} \mathrm{galaxies} \mathrm{in} \mathrm{the} \mathrm{Hydra} \mathrm{I}$ cluster (see Duc et al. 1999, Paper I). Long-slit spectra were obtained along an axis including the nucleus and the brightest H II region in each galaxy. The oxygen abundance was estimated from the emission lines using various methods. All dwarfs host one or several star-forming regions that are, for the most part, located outside the nucleus. We did not find in this survey any extremely metal-deficient objects, but we did observe some galaxies with a surprisingly high metallicity for their luminosity. In a following paper in this series, we will discuss the global properties of the $\mathrm{H}$ I-rich dwarfs in the Hydra I cluster and focus on their dependence on the environment.
\end{abstract}

Key words. galaxies: abundances - galaxies: dwarf - galaxies: clusters: general

\section{Introduction}

Because of their low mass, dwarf galaxies in clusters should be very sensitive to their environment. They appear as ideal laboratories to probe environmental effects such as gravitational interactions (see review by Struck 1999) and ram pressure by the intracluster medium (see review by Kenney 1990). Gas rich dwarfs are particularly attractive since their gas, which usually extends far beyond the optical radius, should be easily stripped in clusters. In this respect, the discovery of $\mathrm{H}$ I-rich dwarf galaxies in H I surveys of nearby clusters (e.g. Cayatte et al. 1990; McMahon 1993; Dickey 1997; Bravo-Alfaro et al. 2000; Valluri et al. 2001) is puzzling. Their survival puts constraints on their distribution in the clusters and/or on the properties of the intracluster medium.

We have collected multi-wavelength data of a complete sample of fifteen gas-rich dwarf galaxies in the Hydra I cluster. All of these have been detected in $\mathrm{HI}$ in a VLA survey that covered seventy percent of the cluster core volume (McMahon 1993; Valluri et al. 2001). Most detections were confirmed using the Nançay single-dish radio telescope. For one Hi source, we did not find any optical counterpart. Our selection criteria, Nançay H I data

\footnotetext{
Send offprint requests to: P.-A. Duc, e-mail: paduc@cea.fr

* Based on observations collected at the European Southern Observatory, La Silla, Chile (ESO No 60.A-0791).
}

and optical/near-infrared imaging results were presented in Duc et al. (1999) (hereafter Paper I). We found that the $\mathrm{H}$ I-selected dwarfs in Hydra show a large diversity in their colour, morphology and structural parameters. They range from blue compact objects to red low surface brightness galaxies. All of them, however, host extended or more compact blue regions where star-formation is probably taking place. In this paper, we present spectrophotometric data on all of the 15 galaxies.

The observations at the ESO $3.6 \mathrm{~m}$ telescope and data-reduction are described in Sect. 1 . The derived spectroscopic properties, distribution of $\mathrm{H}$ II regions, starformation rate, and metallicity are presented in Sect. 2. The overall properties of the galaxies in the Hydra cluster and their link with the environment, including their TullyFisher relation, will be discussed in an associated paper (Paper III).

\section{Observations and data reduction}

Long-slit spectroscopic observations were carried out in 1998 March 4-5 and April 24, with the ESO-3.6 m telescope at the la Silla observatory. The EFOSC 2 instrument was used with the B300 grism and a Loral 2k CCD detector. Its pixel size was $0 . \prime 314 / \mathrm{px}$ and the spectral resolution was $3.3 \AA /$ px. A movable slit with a width of $1^{\prime \prime}$. 2 was selected and positioned on the CCD to ensure a wavelength 
Table 1. Observing log

\begin{tabular}{llccc}
\hline Object & Date & Exposure & Airmass & Weather \\
\hline H1031-2632 & March 05, 1998 & $1 \times 900$ & 1.65 & cloudy \\
H1031-2818 & March 04, 1998 & $3 \times 900$ & 1.14 & fair \\
H1032-2638 & March 05, 1998 & $3 \times 900$ & 1.58 & fair \\
H1032-2722 & March 04, 1998 & $3 \times 900$ & 1.00 & fair \\
H1033-2642 & March 04, 1998 & $3 \times 900$ & 1.02 & fair \\
H1033-2707 & March 04, 1998 & $3 \times 900$ & 1.13 & fair \\
H1033-2722 & March 05, 1998 & $3 \times 900$ & 1.00 & fair \\
H1034-2558 & March 05, 1998 & $1 \times 900$ & 1.05 & fair \\
H1034-2758 & April 24, 1998 & $1 \times 900$ & 1.01 & fair \\
H1035-2502 & March 05, 1998 & $3 \times 300$ & 1.28 & fair \\
H1035-2605 & March 05, 1998 & $3 \times 900$ & 1.10 & fair \\
H1035-2740 & March 04, 1998 & $3 \times 900$ & 1.34 & fair \\
H1035-2756 & March 05, 1998 & $3 \times 900$ & 1.02 & fair \\
H1038-2730 & March 04, 1998 & $1 \times 1200$ & 1.99 & cloudy \\
H1038-2733 & March 04, 1998 & $1 \times 1200$ & 1.73 & fair \\
\hline
\end{tabular}

coverage ranging from $3700 \AA$ to $7000 \AA$. The slit was oriented to include both the nucleus of each galaxy and its bluest knot as determined from our $B-I$ colour maps (Paper I). For a few systems, spectra of companion galaxies were also obtained. Their redshifts are given in Paper I. Ten out of the fifteen galaxies were observed with an airmass below 1.3. For those, differential atmospheric refraction and related slit losses should be minimal (Filippenko 1982). The observations generally consisted of three exposures of $900 \mathrm{~s}$ each. Due to time and weather constraints, the exposure time had to be shortened for some objects; these are indicated in Table 1 together with the observing conditions. Atmospheric conditions were spectroscopic during almost the entire run and photometric during half of it. The average seeing was $0 . \prime 9$.

Data reduction was carried out with the CCDRED and LONGSLIT packages of IRAF, following standard procedures for bias subtraction, flat-fielding and wavelength calibration using HeAr reference spectra. Multiple exposures were combined and cosmic rays deleted during that process. For the flux calibration, several spectroscopic standard stars were observed during the two nights. Spectra were obtained with both the same movable 1". 2 slit as used for the galaxy exposures, and a wider but fixed $5^{\prime \prime}$ slit which contained all the flux but unfortunately resulted in a slightly different wavelength coverage from the first slit. For a given star, the response curve resulting from the 1 .' 2 spectra was scaled to match that from the $5^{\prime \prime}$ slit in the common wavelength domain. All consistent curves were then averaged while the deviant ones were excluded.

Several spectra were extracted with the APEXTRACT package from each $2 \mathrm{D}$ spectrum: one integrated over the entire galaxy along the slit, one of its nucleus, if present, and one for each $\mathrm{H}$ II region. Furthermore, the $\mathrm{H}_{\alpha}$ line was measured automatically along the slit with the FITPROFS command. At each position, fluxes were integrated over 5 pixels $\left(1.5^{\prime \prime}\right)$. Velocity curves were derived from these data at positions where the fluxes have a sufficiently high signal-to-noise ratio.
In Figs. 1 to 14, for each object we show the slit orientation superimposed on a $B$-band image (see Paper I), the $2 \mathrm{D}$ spectrum in the $\mathrm{H}_{\alpha}$ region, the corresponding velocity curve and the spectrum integrated over the whole galaxy.

All lines fluxes and associated errors were measured manually with SPLOT. The errors take into account the gain of the detector and the measured rms of the continuum next to the lines. Data were corrected for extinction using the formula:

$\frac{I(\lambda)}{I\left(\mathrm{H}_{\beta}\right)}=\frac{F(\lambda)}{F\left(\mathrm{H}_{\beta}\right)} * 10^{c * f(\lambda)}$

where $F(\lambda)$ is the observed line flux, $f(\lambda)$ the reddening function taken from Torres-Peimbert et al. (1989) and $c$ the logarithmic reddening correction at $\mathrm{H}_{\beta}$ obtained from a constant $\mathrm{H}_{\alpha} / \mathrm{H}_{\beta}$ Balmer decrement of 2.85. In a few cases, an $\mathrm{H}_{\beta}$ emission line from $\mathrm{H}$ II regions is seen superimposed on $\mathrm{H}_{\beta}$ absorption from the underlying stellar component; in these cases, the absorption line, which generally has broader wings, might contaminate the flux of the emission line. We performed a first order correction of this effect by deblending the two lines with two Gaussians, a rather rough method due to the lack of spectral resolution. In order to estimate its accuracy, we fitted two spectra of H II regions showing strong Balmer absorption lines, those of H1031-2818 and H1035-2605, with a combination of stellar spectra of different spectral classes. We used, in the library of Jacoby et al. (1984), A, F and $\mathrm{G}$ main sequence stars and $\mathrm{B}$ giants. The spectra had to be dereddenned by $A_{\mathrm{V}}=0.6 \mathrm{mag}$ in order to reproduce the continuum and the absorption lines $(\mathrm{Ca} \mathrm{H}+\mathrm{K}, \mathrm{H} 8$, H9, H10, G-band, MgI). We then measured the fluxes of the Balmer emission lines after subtraction of the model stellar spectrum. We found that these $\mathrm{H}_{\beta}$ fluxes differ by less than $10 \%$ from the $\mathrm{H}_{\beta}$ fluxes measured directly in the spectra, i.e. within the instrumental errors. For the $\mathrm{H}_{\alpha}$ line, which is less affected by stellar absorption, the error is less than $3 \%$. Without deblending the lines, the error would have been about 20\%. Therefore, the deblending procedure provides a reasonable correction for underlying stellar absorption, while not introducing a large error.

Tables 2-4 list the flux values of the $\mathrm{H}_{\beta}$ line and the observed and extinction corrected fluxes relative to $\mathrm{H}_{\beta}$ of the principal emission lines.

Only the data corresponding to the brightest H II regions and having the highest signal to noise ratios are listed in Tables $2-4$. For those, the contamination of the emission lines by underlying stellar absorption should be minimal. Values followed by ":" are very uncertain and no errors are given for them. The $\mathrm{H}_{\alpha}$ and $[\mathrm{NII}]_{\lambda 6548}$ lines have been deblended. Notwithstanding, the fluxes of the latter line have a high uncertainty and are not listed in the table. Because of the low signal to noise of the $[\mathrm{SII}]_{\lambda 6717}$ and $[\mathrm{SII}]_{\lambda 6731}$ lines in some spectra, they could not be deblended. In such cases (identified in the table by “..."), the combined fluxes are given. 

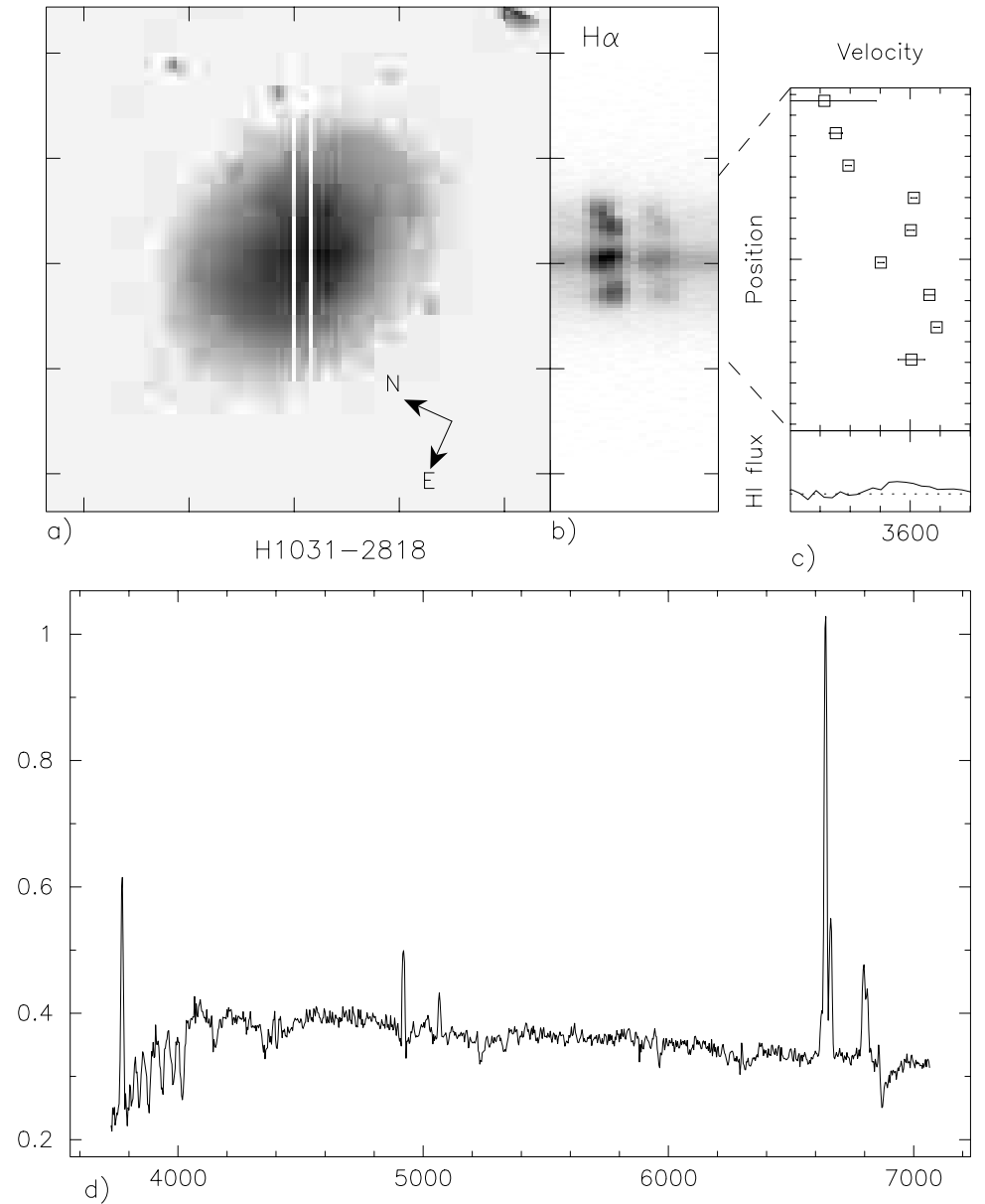

Fig. 1. H1031-2818: a) The position of the long-slit is superimposed on the $B$ band image of the galaxy. The orientation on the sky is indicated by the arrows. The tickmarks are separated by $10^{\prime \prime}(2.2 \mathrm{kpc})$. b) $2 \mathrm{D}$ spectrum in the $\mathrm{H}_{\alpha}+[\mathrm{NII}]$ region. The dispersion is along the lines. The spatial axis is the same as in Fig. a). c) Top: $\mathrm{H}_{\alpha}$ velocity curve along the slit position. The optical heliocentric velocities ( $x$-axis) are in $\mathrm{km} \mathrm{s}^{-1}$. Along the spatial axis ( $y$-axis), the major tickmarks are separated by $10^{\prime \prime}$ and the minor ones by $1^{\prime \prime}$. Bottom: H I spectrum obtained at Nançay. The velocity scale is the same as for the optical velocity curve. For galaxies that have not been re-observed at Nançay, the VLA Hi line width is indicated instead. d) Optical long-slit spectrum integrated over the whole galaxy. The dispersion axis is in $\AA$. The fluxes are in $10^{-15} \mathrm{erg} \mathrm{cm}^{-2} \mathrm{~s}^{-1} \AA^{-1}$
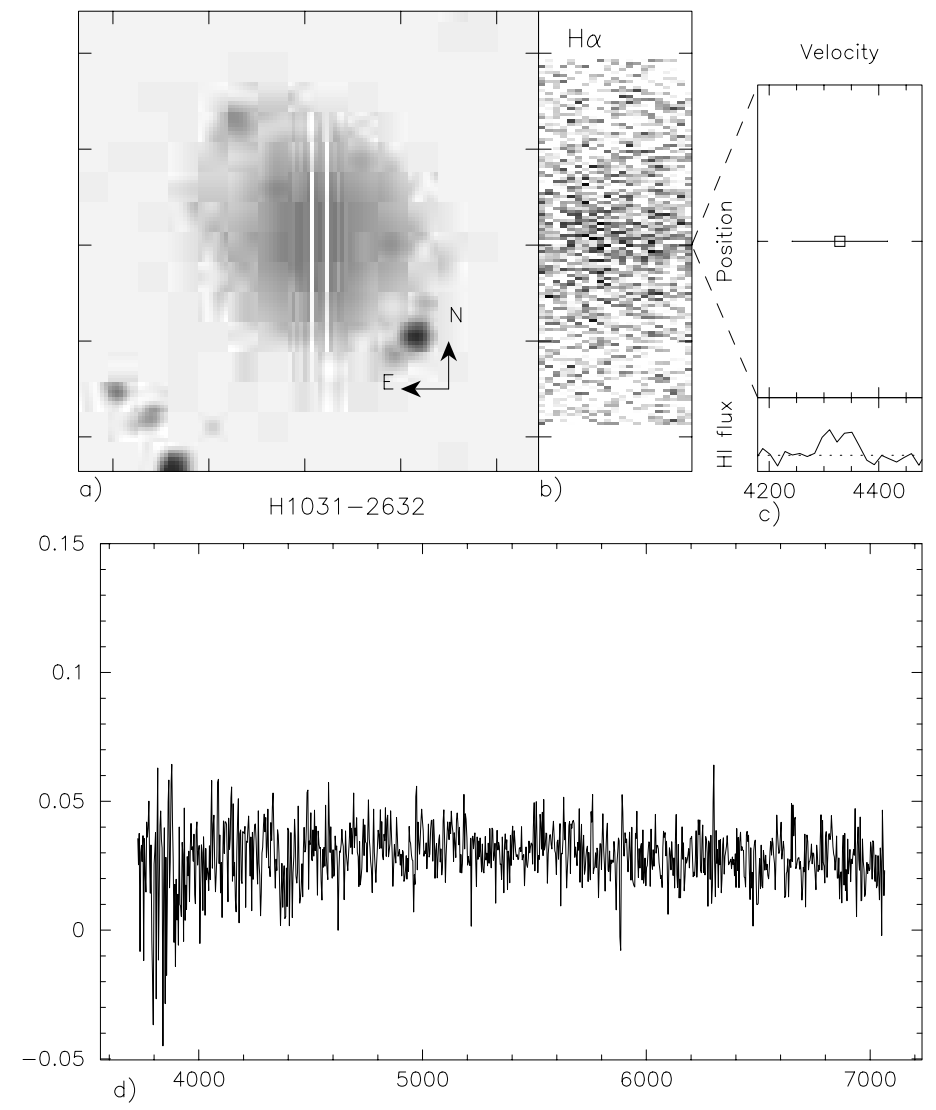

Fig. 2. H1031-2632 

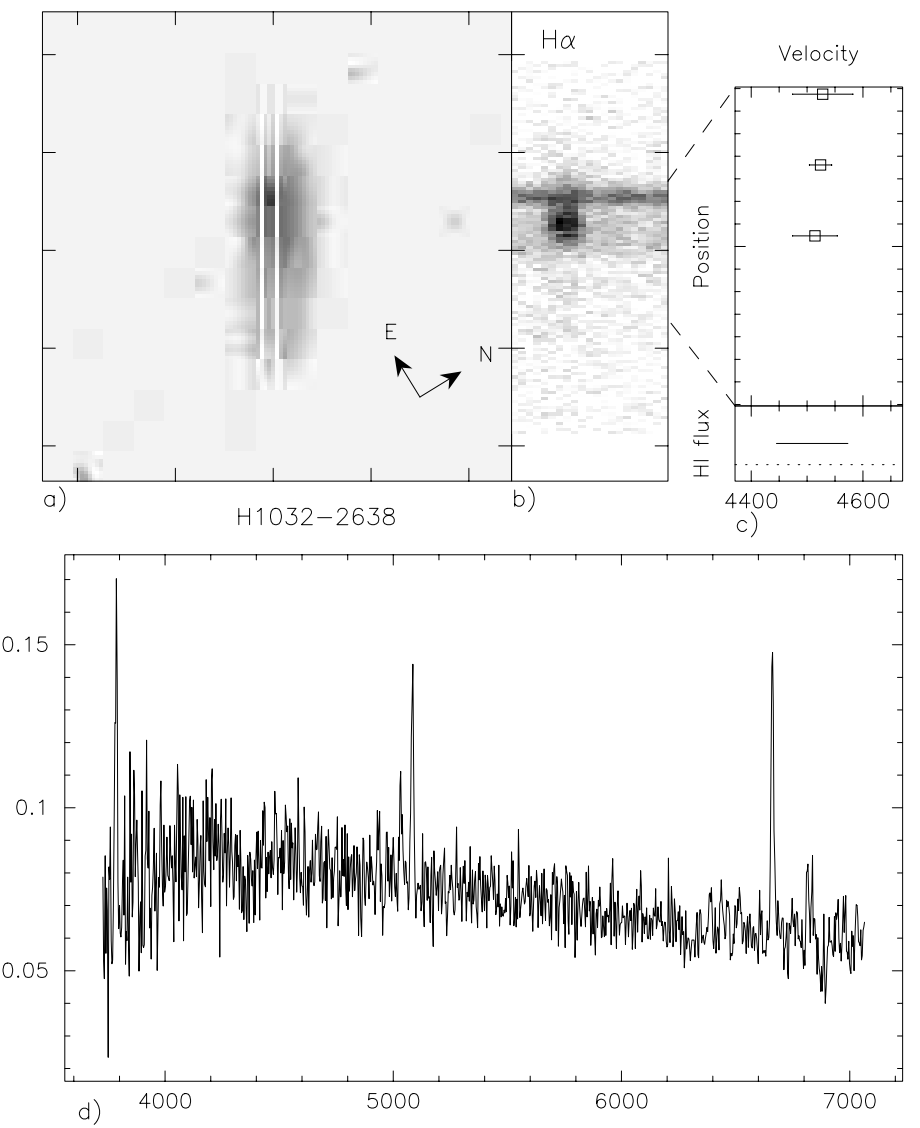

Fig. 3. H1032-2638
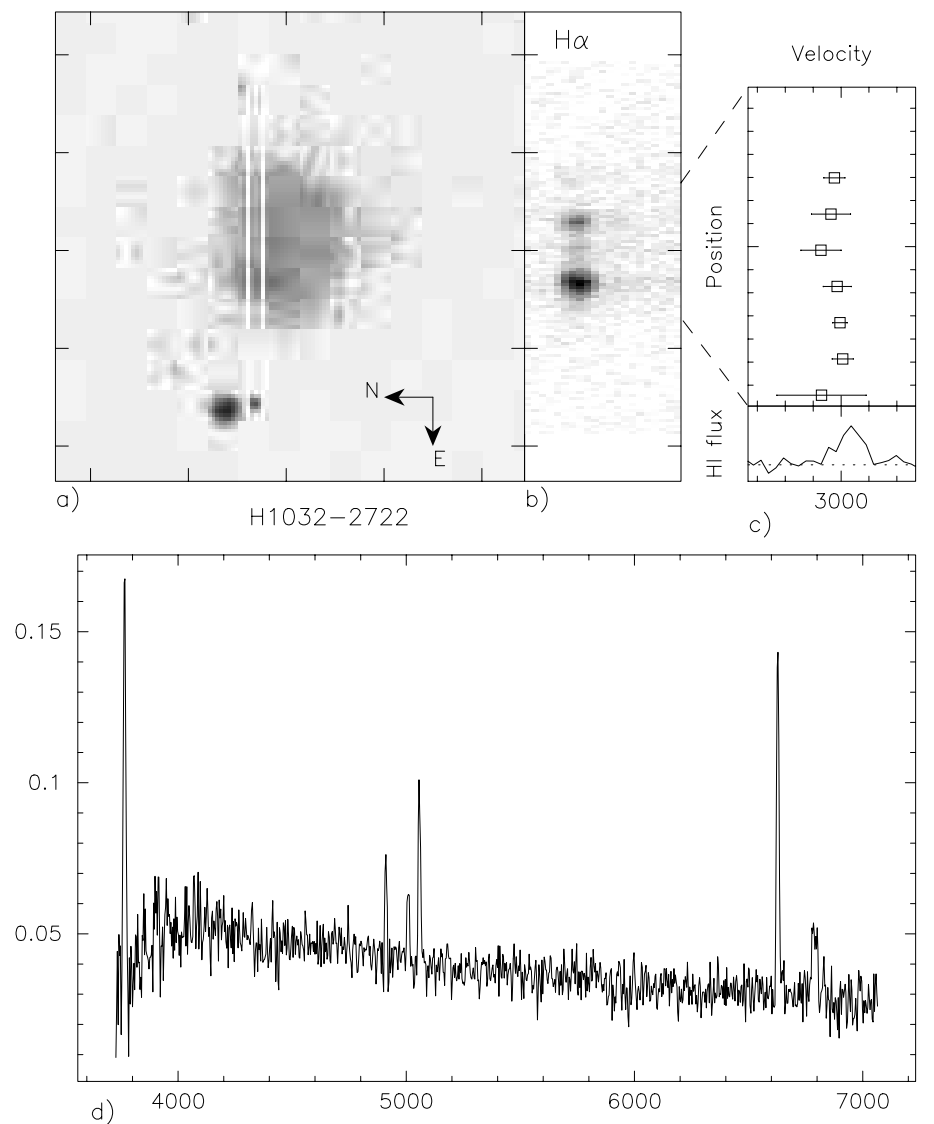

Fig. 4. H1032-2722 
P.-A. Duc et al.: H I-rich dwarf galaxies in the Hydra I cluster. II.
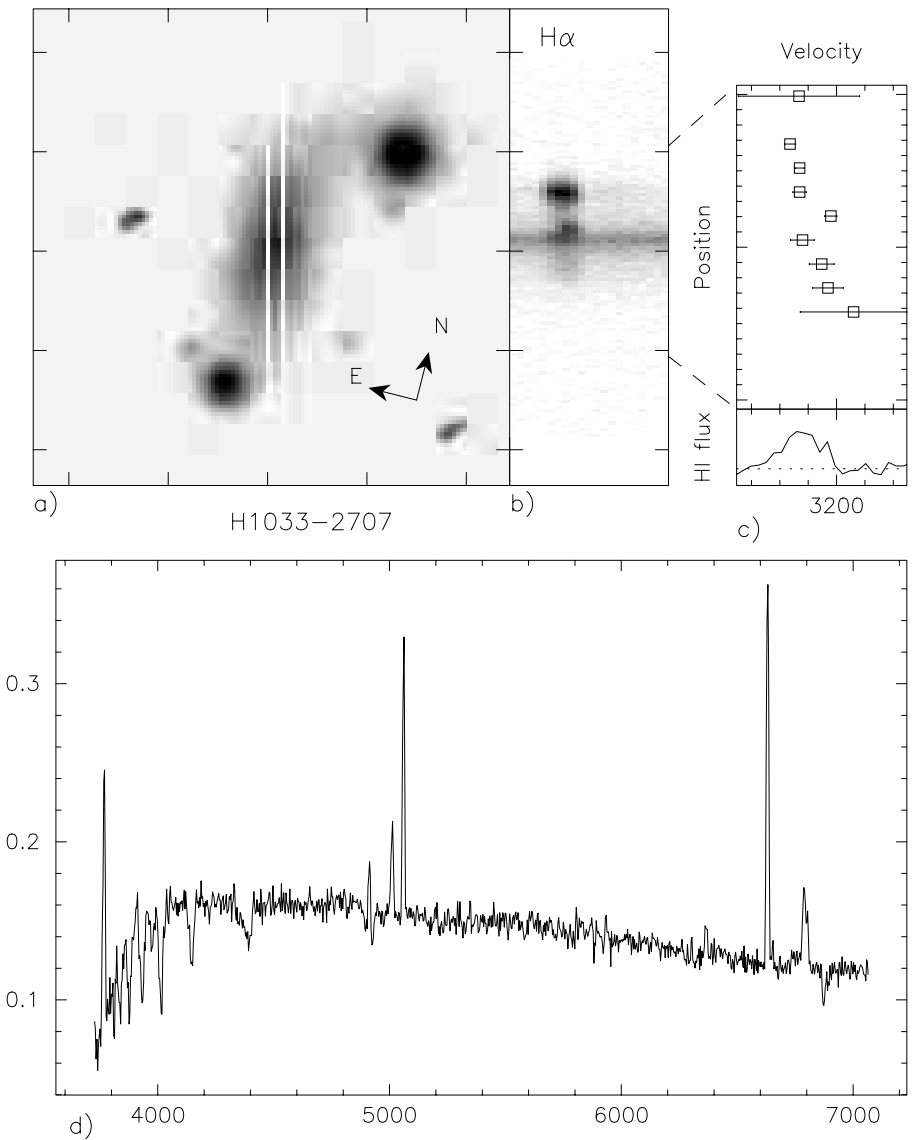

Fig. 5. H1033-2707
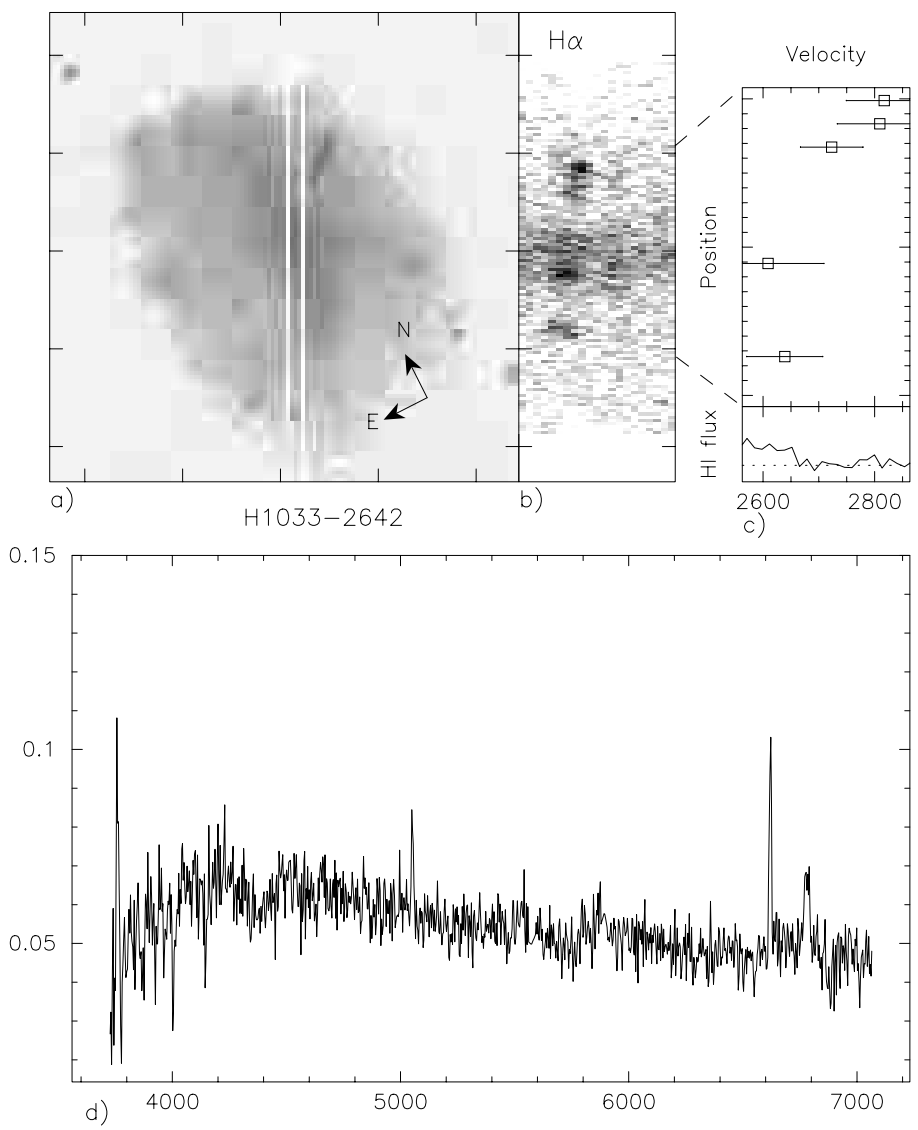

Fig. 6. H1033-2642 

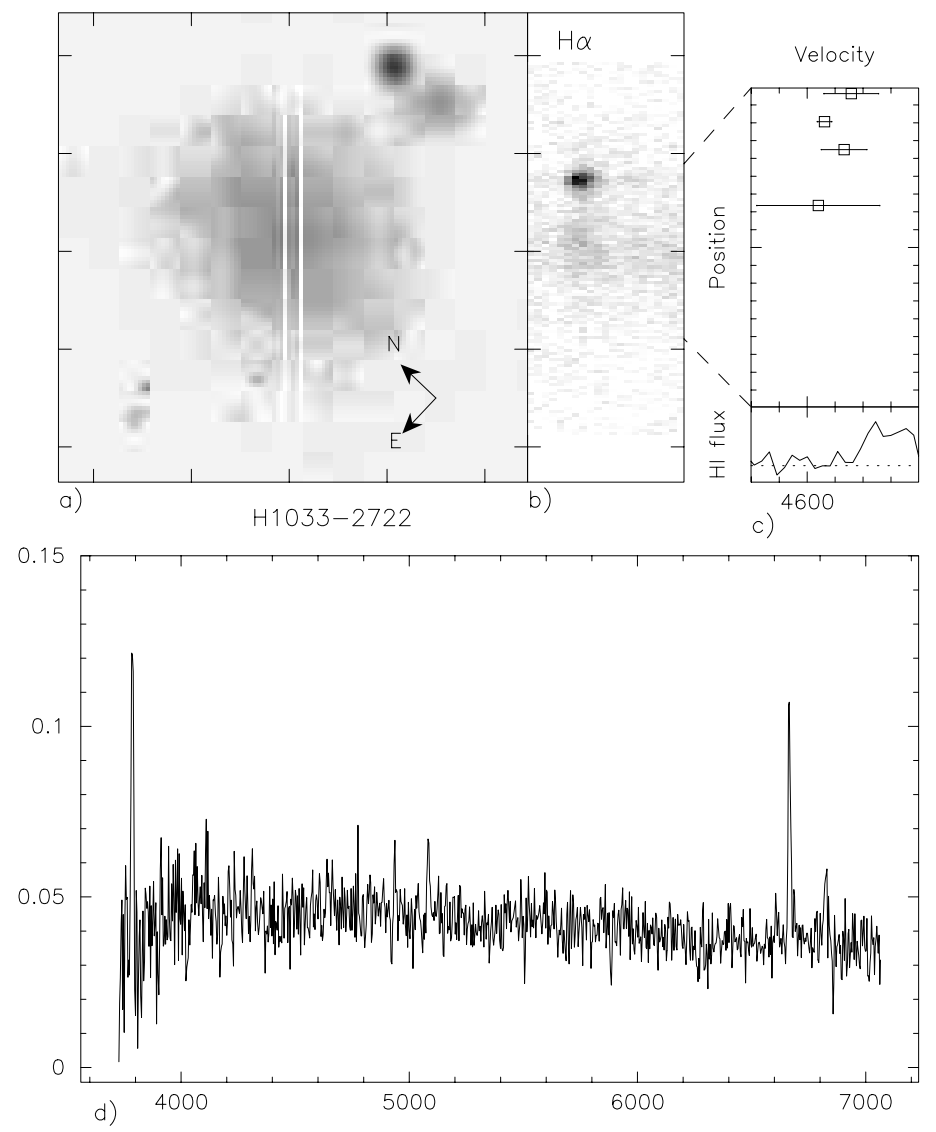

Fig. 7. H1033-2722
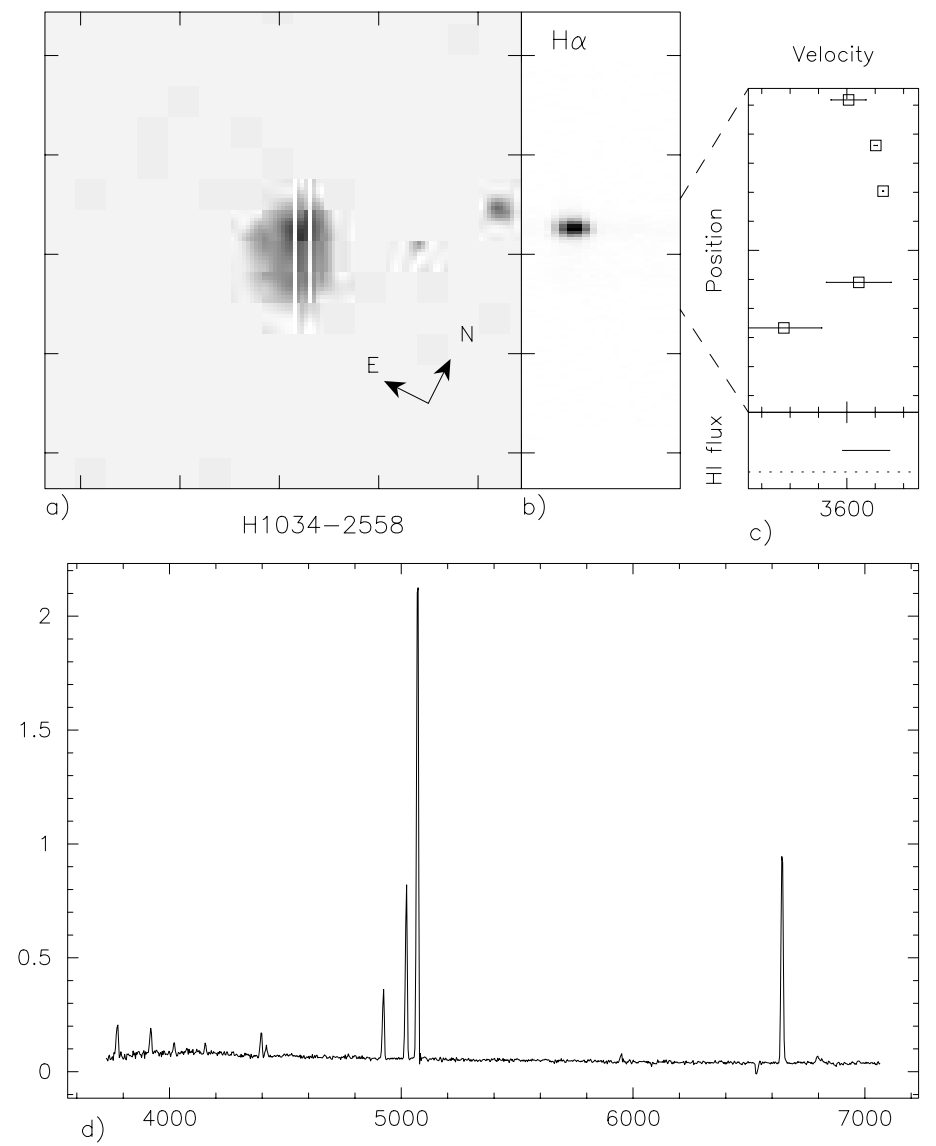

Fig. 8. H1034-2558 

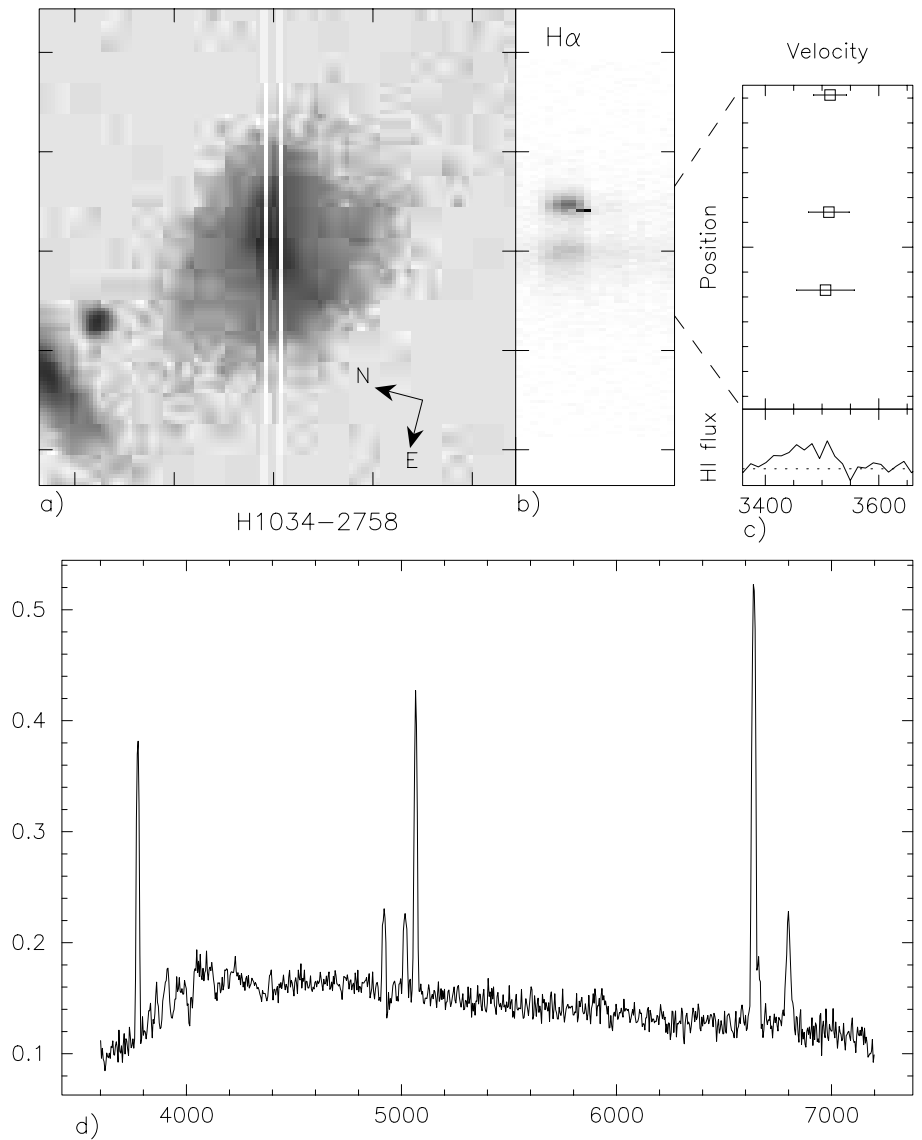

Fig. 9. H1034-2758
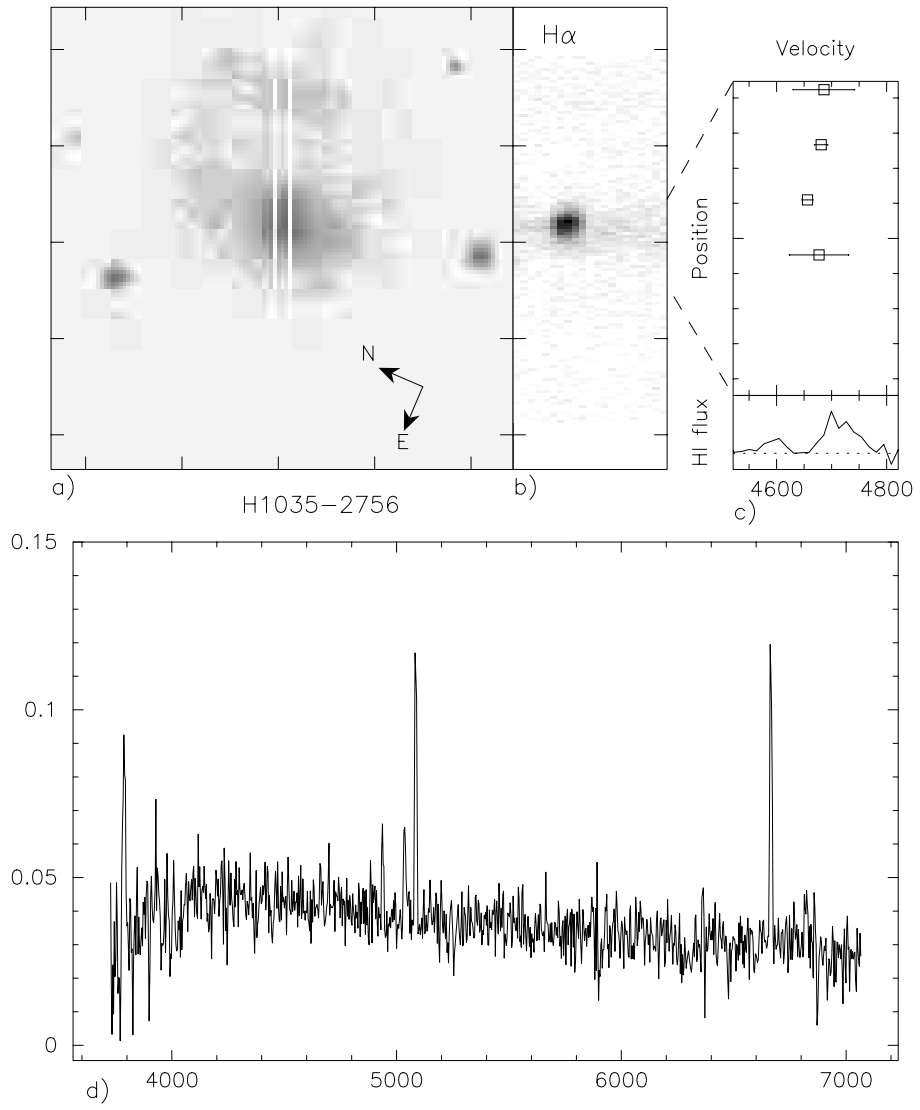

Fig. 10. H1035-2756 

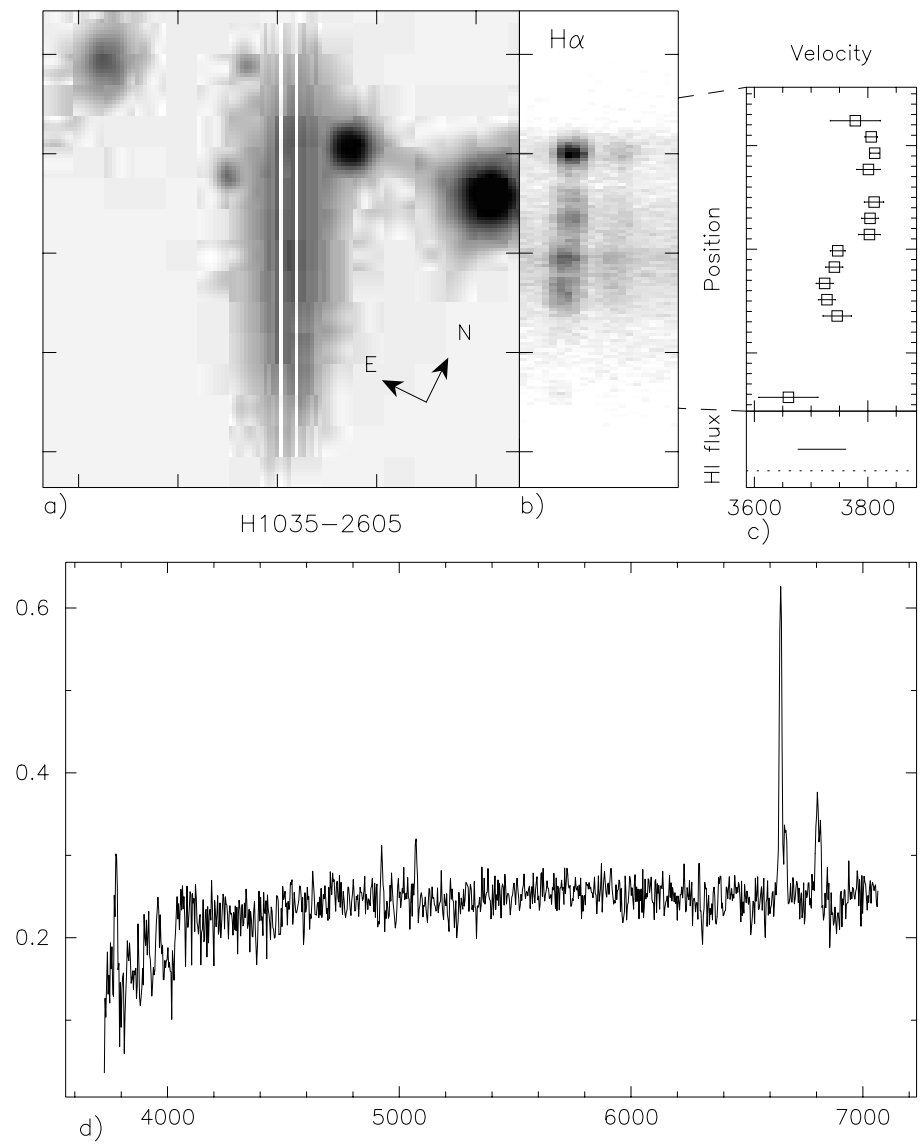

Fig. 11. H1035-2605
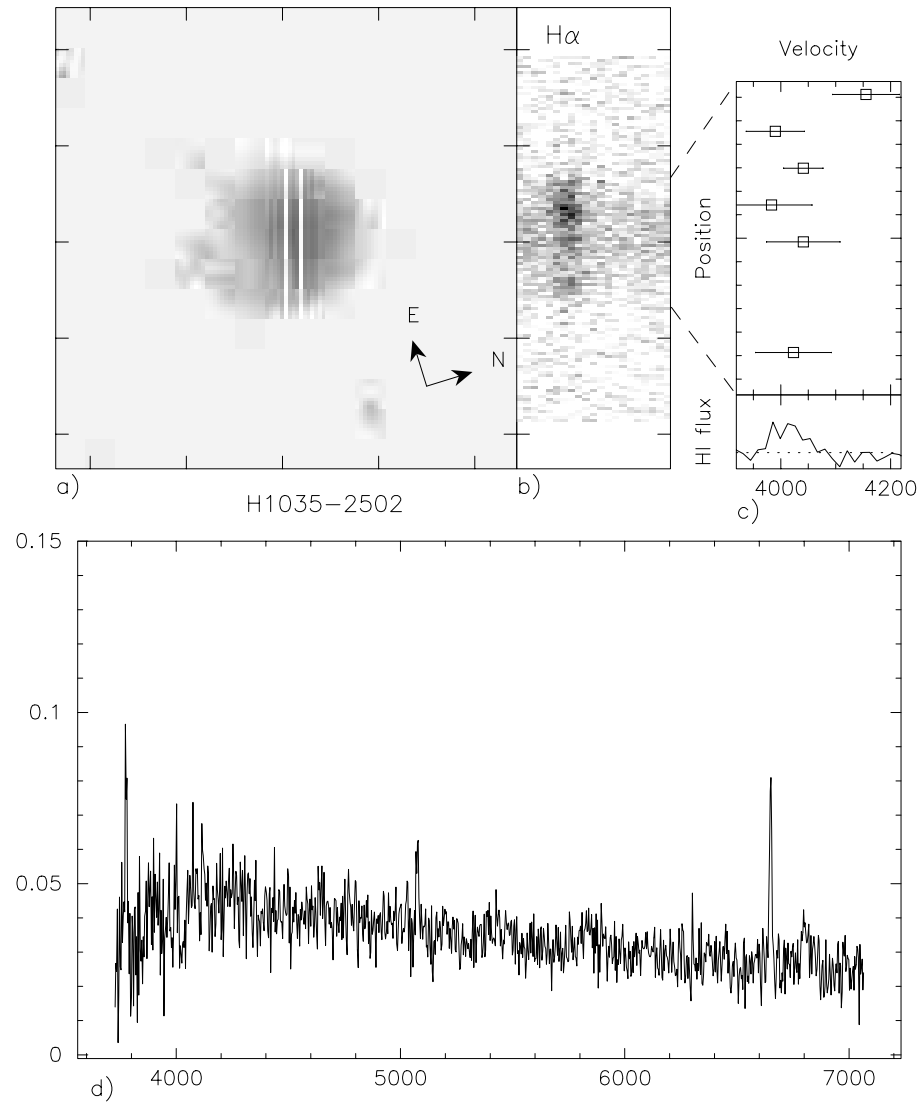

Fig. 12. H1035-2502 

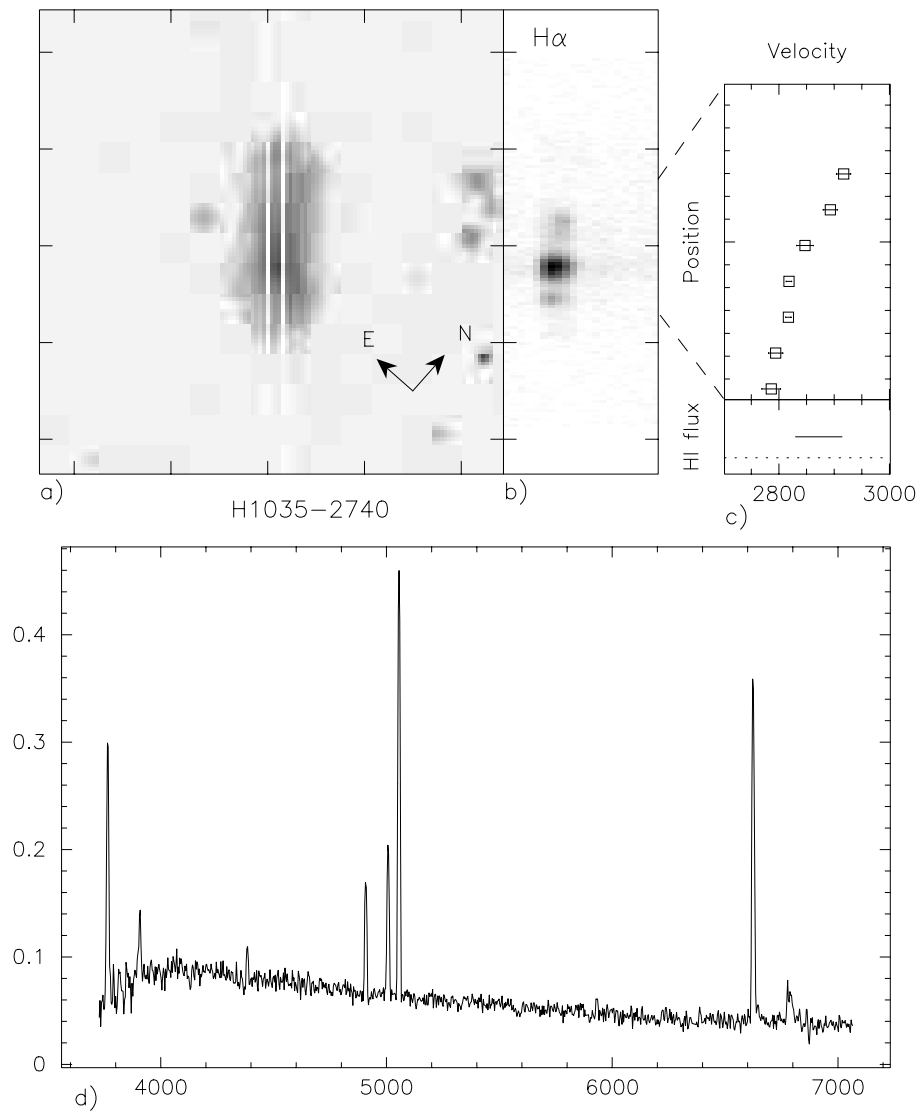

Fig. 13. H1035-2740
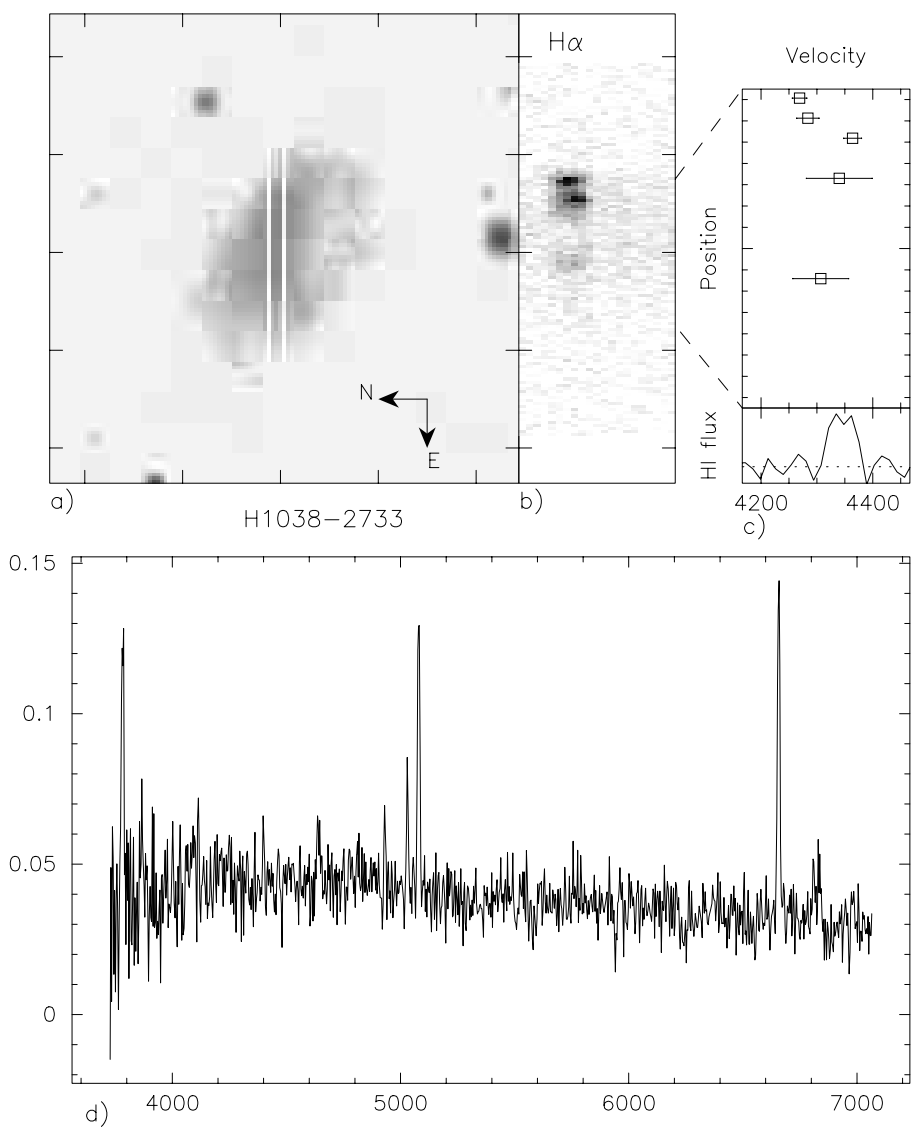

Fig. 14. H1038-2733 

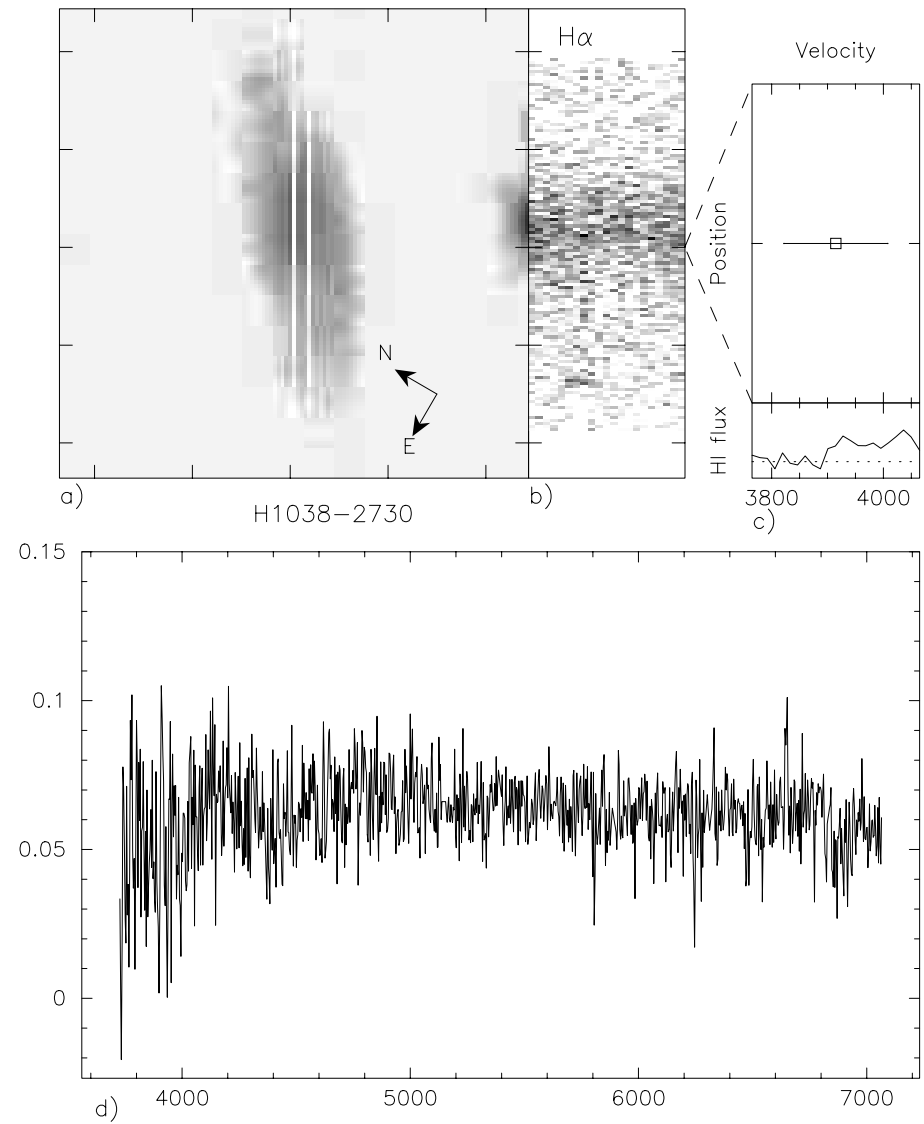

Fig. 15. H1038-2730

Table 2. Spectrophotometric data of the brightest H II regions

\begin{tabular}{|c|c|c|c|c|c|c|}
\hline & & H1031-2818 & H1032-2638 & H1032-2722 & H1033-2642 & H1033-2707 \\
\hline [OII] & 3727. & $196 \pm 12,259 \pm 43$ & $338 \pm 46,417 \pm 127$ & $407 \pm 28,501 \pm 70$ & $1020 \pm 320,2920 \pm 1078$ & $224 \pm 10,307 \pm 33$ \\
\hline $\mathrm{H}_{\delta}$ & 4101.7 & - & - & - & - & - \\
\hline $\mathrm{H}_{\gamma}$ & 4340.5 & $24 \pm 3,27 \pm 15$ & - & $25 \pm 4,27 \pm 20$ & - & $32 \pm 3,36 \pm 12$ \\
\hline [OIII] & 4363.2 & 10:, 11 & - & 8:, 9 & 95:, 148 & - \\
\hline $\mathrm{H}_{\beta}$ & 4861.3 & $100 \pm 8,100 \pm 8$ & $100 \pm 20,100 \pm 20$ & $100 \pm 11,100 \pm 11$ & $100 \pm 51,100 \pm 51$ & $100 \pm 5,100 \pm 5$ \\
\hline [OIII] & 4958.9 & $21 \pm 4,20 \pm 6$ & $107 \pm 20,106 \pm 24$ & $61 \pm 8,61 \pm 10$ & - & $90 \pm 5,88 \pm 6$ \\
\hline [OIII] & 5006.7 & $34 \pm 4,33 \pm 6$ & $311 \pm 42,306 \pm 47$ & $160 \pm 14,157 \pm 17$ & $545 \pm 187,496 \pm 185$ & $263 \pm 10,256 \pm 11$ \\
\hline $\mathrm{H}_{\alpha}$ & 6562.8 & $384 \pm 19,285 \pm 43$ & $357 \pm 47,285 \pm 112$ & $356 \pm 24,285 \pm 57$ & $872 \pm 263,285 \pm 258$ & $398 \pm 14,285 \pm 31$ \\
\hline$[\mathrm{NII}]$ & 6583.4 & $114 \pm 7,84 \pm 34$ & $21 \pm 7,17 \pm 81$ & $17 \pm 4,13 \pm 42$ & 86:, 28 & $25 \pm 3,18 \pm 23$ \\
\hline [SII] & 6716.4 & $81 \pm 6,58 \pm 36$ & $62 \pm 13,49 \pm 92$ & $73 \pm 8,57 \pm 48$ & $377 \pm 165,110 \pm 238$ & $63 \pm 5,43 \pm 26$ \\
\hline [SII] & 6730.8 & $55 \pm 5,39 \pm 35$ & $44 \pm 12,35 \pm 92$ & $56:, 44$ & 236:, 68 & $41 \pm 3,28 \pm 25$ \\
\hline \multirow{3}{*}{\multicolumn{2}{|c|}{$\begin{array}{l}\text { Reddening Factor (c) } \\
\mathrm{H}_{\beta} \text { flux }\left(10^{-15} \mathrm{erg} \mathrm{cm}^{-2} \mathrm{~s}^{-1}\right) \\
\mathrm{H}_{\beta} \text { equivalent width }(\AA)\end{array}$}} & $0.39 \pm 0.13$ & $0.29 \pm 0.33$ & $0.29 \pm 0.17$ & $1.45 \pm 0.78$ & $0.43 \pm 0.09$ \\
\hline & & $0.64 \pm 0.05$ & $0.20 \pm 0.04$ & $0.25 \pm 0.03$ & $0.02 \pm 0.01$ & $0.41 \pm 0.02$ \\
\hline & & $10 \pm 1$ & $10 \pm 2$ & $23 \pm 2$ & $2 \pm 1$ & $19 \pm 1$ \\
\hline
\end{tabular}

\section{Results}

\subsection{Optical and $\mathrm{HI}$ velocities}

The mean optical velocities, presented in Table 5, were derived using several emission lines present in the integrated spectra. A higher weight was given to the $\mathrm{H}_{\alpha}$ line which generally has the highest signal-to-noise ratio. The H I ve- locities were determined from the Nançay spectra, when available, or otherwise from the VLA data (see Paper I). The uncertainties in the central H I velocities were estimated following Fouqué et al. (1990).

In general, the agreement between the global optical and $\mathrm{HI}$ velocities is quite good. The average of the $\mathrm{HI}$ optical velocity differences is $+15 \mathrm{~km} \mathrm{~s}^{-1}$, which is within 
Table 3. Spectrophotometric data of the brightest H II regions

\begin{tabular}{|c|c|c|c|c|c|c|}
\hline & & H1033-2722 & H1034-2558 & H1034-2758 & H1035-2502 & H1035-2605 \\
\hline$[\mathrm{OII}]$ & 3727. & $314 \pm 24,314 \pm 59$ & $49 \pm 2,62 \pm 9$ & $236 \pm 8,481 \pm 32$ & $824 \pm 207,1768 \pm 581$ & $227 \pm 22,349 \pm 78$ \\
\hline $\mathrm{H}_{\delta}$ & 4101.7 & - & $14 \pm 1,17 \pm 5$ & 13:, 20 & - & - \\
\hline $\mathrm{H}_{\gamma}$ & 4340.5 & $36:, 36$ & $37 \pm 1,41 \pm 4$ & $36 \pm 3,49 \pm 11$ & - & $20:, 24$ \\
\hline [OIII] & 4363.2 & - & $13 \pm 1,14 \pm 4$ & - & - & - \\
\hline $\mathrm{H}_{\beta}$ & 4861.3 & $100 \pm 11,100 \pm 11$ & $100 \pm 2,100 \pm 2$ & $100 \pm 5,100 \pm 5$ & $100 \pm 40,100 \pm 40$ & $100 \pm 14,100 \pm 14$ \\
\hline [OIII $]$ & 4958.9 & $58 \pm 9,58 \pm 11$ & $253 \pm 3,249 \pm 4$ & $119 \pm 5,114 \pm 6$ & - & $31 \pm 7,30 \pm 9$ \\
\hline [OIII $]$ & 5006.7 & $129 \pm 12,129 \pm 15$ & $763 \pm 9,746 \pm 9$ & $387 \pm 11,363 \pm 12$ & $149 \pm 55,139 \pm 64$ & $107 \pm 12,103 \pm 16$ \\
\hline $\mathrm{H}_{\alpha}$ & 6562.8 & $265 \pm 19,265 \pm 56$ & $371 \pm 5,285 \pm 11$ & $608 \pm 19,285 \pm 27$ & $642 \pm 164,285 \pm 218$ & $450 \pm 36,285 \pm 69$ \\
\hline$[\mathrm{NII}]$ & 6583.4 & $33 \pm 5,33 \pm 43$ & $3:, 2$ & $31 \pm 3,14 \pm 20$ & $35:, 15$ & $92 \pm 10,58 \pm 53$ \\
\hline [SII] & 6716.4 & $57 \pm 9,57 \pm 50$ & $12 \pm 1,9 \pm 8$ & $115 \pm 6,50 \pm 22$ & $493 \pm 232,202 \pm 255$ & $117 \pm 15,71 \pm 60$ \\
\hline [SII] & 6730.8 & $\ldots$ & $8 \pm 1,6 \pm 8$ & $\ldots$ & $\ldots$ & $78 \pm 9,47 \pm 57$ \\
\hline \multicolumn{2}{|c|}{ Reddening Factor (c) } & $0.0:$ & $0.34 \pm 0.03$ & $0.98 \pm 0.08$ & $1.05 \pm 0.66$ & $0.59 \pm 0.21$ \\
\hline \multicolumn{2}{|c|}{$\mathrm{H}_{\beta}$ flux $\left(10^{-15} \mathrm{erg} \mathrm{cm}^{-2} \mathrm{~s}^{-1}\right)$} & $0.20 \pm 0.02$ & $2.79 \pm 0.05$ & $0.87 \pm 0.04$ & $0.04 \pm 0.02$ & $0.36 \pm 0.05$ \\
\hline \multicolumn{2}{|c|}{$\mathrm{H}_{\beta}$ equivalent width $(\AA)$} & $36 \pm 4$ & $74 \pm 1$ & $23 \pm 1$ & $3 \pm 1$ & $8 \pm 1$ \\
\hline
\end{tabular}

Table 4. Spectrophotometric data of the brightest H II regions

\begin{tabular}{lcccc}
\hline & H1035-2740 & H1035-2756 & H1038-2733 \\
\hline$[\mathrm{OII}]$ & 3727. & $179 \pm 6,197 \pm 19$ & $348 \pm 43,530 \pm 132$ & $259 \pm 24,315 \pm 79$ \\
$\mathrm{H}_{\delta}$ & 4101.7 & - & - & - \\
$\mathrm{H}_{\gamma}$ & 4340.5 & $34 \pm 2,35 \pm 8$ & - & $43 \pm 7,47 \pm 29$ \\
{$[\mathrm{OIII}]$} & 4363.2 & $4:, 5$ & - & - \\
$\mathrm{H}_{\beta}$ & 4861.3 & $100 \pm 4,100 \pm 4$ & $100 \pm 19,100 \pm 19$ & $100 \pm 14,100 \pm 14$ \\
{$[\mathrm{OIII}]$} & 4958.9 & $143 \pm 5,142 \pm 5$ & $150 \pm 21,146 \pm 25$ & $148 \pm 16,146 \pm 19$ \\
{$[\mathrm{OIII}]$} & 5006.7 & $404 \pm 10,401 \pm 11$ & $379 \pm 45,365 \pm 49$ & $338 \pm 31,332 \pm 35$ \\
$\mathrm{H}_{\alpha}$ & 6562.8 & $315 \pm 8,285 \pm 21$ & $446 \pm 56,285 \pm 107$ & $351 \pm 33,285 \pm 79$ \\
{$[\mathrm{NII}]$} & 6583.4 & $7 \pm 1,6 \pm 15$ & $29:, 18$ & $31:, 25$ \\
{$[\mathrm{SII}]$} & 6716.4 & $24 \pm 2,21 \pm 17$ & $72:, 44$ & $18:, 14$ \\
{$[\mathrm{SII}]$} & 6730.8 & $22 \pm 2,20 \pm 17$ & $49:, 30$ & $25:, 20$ \\
\hline Reddening Factor $(\mathrm{c})$ & $0.13 \pm 0.06$ & $0.58 \pm 0.32$ & $0.27 \pm 0.24$ \\
$\mathrm{H}_{\beta}$ flux $\left(10^{-15}\right.$ erg cm $\left.{ }^{-2} \mathrm{~s}^{-1}\right)$ & $0.83 \pm 0.03$ & $0.25 \pm 0.05$ & $0.18 \pm 0.02$ \\
$\mathrm{H}_{\beta}$ equivalent width $(\AA)$ & $36 \pm 1$ & $8 \pm 2$ & $24 \pm 3$ \\
\hline
\end{tabular}

the errors. Therefore, we do not find any systematic shift between the $\mathrm{H}$ I and optical velocities (see Fig. 16). This is a further confirmation that all the $\mathrm{H}$ I detections reported in Paper I are secure. A direct comparison of the optical velocity curve along the slit and the $\mathrm{H}$ i line profile is presented in Figs. 1c to 14c. Though the slit was aligned along the major optical axis for most edge-on systems, this was not always the case for less inclined objects. Studying a sample of blue compact dwarf galaxies mapped with the VLA, van Zee et al. (2001) note that the kinematical axis of a galaxy infered from the H I 2D map can differ from the optical major axis, and may even be perpendicular to it. Therefore a mass estimate of the galaxies in our sample based on the 1D optical spectrum only could be unreliable, or, in principle, even completely untrustworthy in extreme cases. The integrated H s spectrum obtained at Nançay is a better tool for estimating rotational velocities. The latter will be used in Paper III to examine the Tully-Fisher relation. 
Table 5. Velocities

\begin{tabular}{lll}
\hline Object & $\begin{array}{l}\text { Vel(HI) } \\
\mathrm{km} \mathrm{s}^{-1}\end{array}$ & $\begin{array}{l}\text { Vel(opt) } \\
\mathrm{km} \mathrm{s}^{-1}\end{array}$ \\
\hline H1031-2818 & $3590 \pm 11$ & $3558 \pm 3$ \\
H1031-2632 & $4326 \pm 5$ & $4333:$ \\
H1032-2638 & $4509 \pm 16$ & $4513 \pm 16$ \\
H1032-2722 & $3021 \pm 10$ & $2977 \pm 8$ \\
H1033-2707 & $3147 \pm 12$ & $3134 \pm 6$ \\
H1033-2642 & $2599 \pm 8$ & $2677 \pm 26$ \\
H1033-2722 & $4747 \pm 7$ & $4648 \pm 25$ \\
H1034-2558 & $3634 \pm 33$ & $3689 \pm 1$ \\
H1034-2758 & $3468 \pm 14$ & $3463 \pm 5$ \\
H1035-2756 & $4715 \pm 9$ & $4658 \pm 19$ \\
H1035-2605 & $3719 \pm 22$ & $3792 \pm 9$ \\
H1035-2502 & $4008 \pm 13$ & $4026 \pm 22$ \\
H1035-2740 & $2872 \pm 20$ & $2829 \pm 3$ \\
H1038-2733 & $4346 \pm 3$ & $4310 \pm 13$ \\
H1038-2730 & $4037 \pm 8$ & $3919 \pm 56$ \\
\hline
\end{tabular}

\subsection{Distribution, luminosity and strength of HII regions}

Our long-slit spectra reveal that almost all H I-rich dwarf galaxies in Hydra contain star-forming regions. The two galaxies with very weak $\mathrm{H}_{\alpha}$ detection, H1031-2632 and H1038-2730, have been observed under poor weather conditions and at a high airmass. Ten out of the fifteen galaxies $(65 \%)$ show more than one HII region, and four of these $(25 \%)$ exhibit star formation along their whole optical disk. In nine galaxies $(60 \%)$, the brightest $\mathrm{H}$ II region is off-center. Note that the slit orientations are such that a minimum number of blue knots, i.e. H II regions candidates, has been missed (see Figs. 1 to 14 and Figs. 14 to 28 in Paper I).

From the integrated $\mathrm{H}_{\alpha}$ emission along the slits, we determined lower limits for the total $\mathrm{H}_{\alpha}$ luminosities, as listed in Table 6. All values were corrected for Galactic extinction only, as the determination of internal extinction from the Balmer decrement is quite unreliable for integrated spectra in which the underlying stellar contribution is significant. The importance of the stellar absorption features may be estimated from the equivalent width of the $\mathrm{H}_{\delta}$ line (Col. 2 in Table 6 ). This line is indeed only weakly affected by the emission from the ionised gas, with the notable exception of the starburst galaxy H1034-2558. Also listed in the table are the equivalent widths of the $[\mathrm{OII}]_{\lambda 3727}$ and $\mathrm{H}_{\beta}$ emission lines. Histograms of the $\mathrm{H}_{\alpha}$ luminosity measured along the integrated long-slit spectra and of the individual $\mathrm{H}$ II regions are shown in Fig. 17 with, respectively, solid and dashed lines. The brightest $\mathrm{H}$ II re-

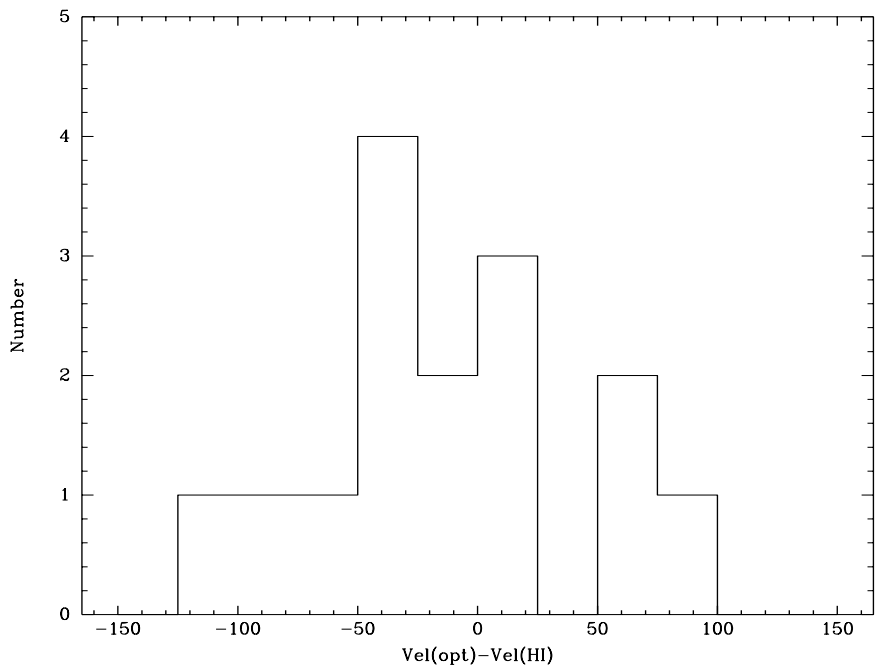

Fig. 16. Histogram of the difference between the optical and H I velocity

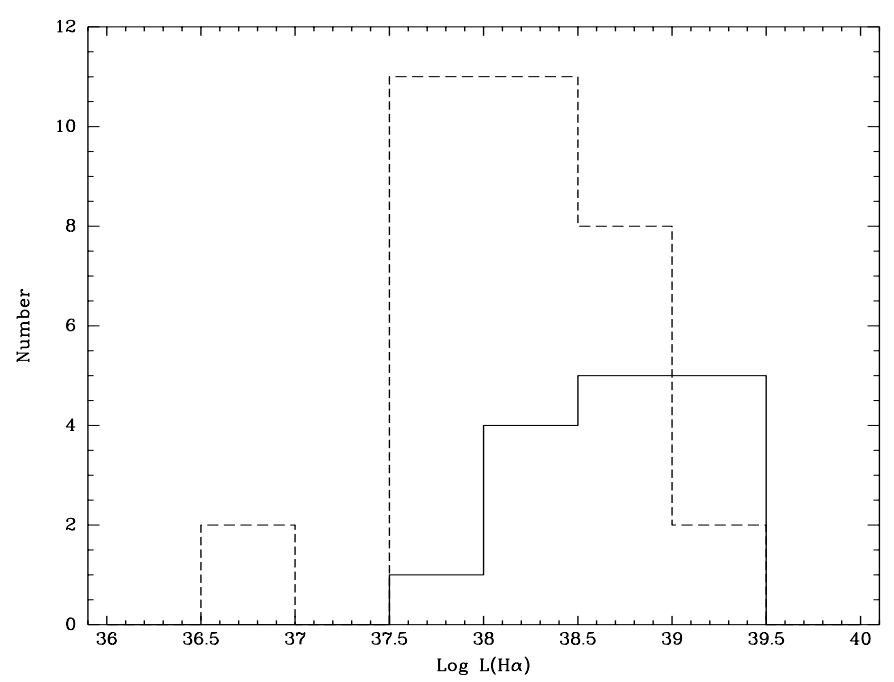

Fig. 17. Histogram of the $H_{\alpha}$ luminosity. The distribution of $L\left(\mathrm{H}_{\alpha}\right)$ corresponding to the integrated emission and individual $\mathrm{H}$ II regions are shown with resp. plain lines and dashed lines

gions have $\mathrm{H}_{\alpha}$ luminosities comparable to 30 Doradus in the LMC $\left(\log \left(L\left(\mathrm{H}_{\alpha}\right)=39.8 ;\right.\right.$ Kennicutt \& Hodge 1986). Lower limits of the star formation rate, estimated from $\mathrm{H}_{\alpha}$ using the calibration of Kennicutt (1998), are on average $0.007 M_{\odot} / \mathrm{yr}$ and reach $0.025 M_{\odot} / \mathrm{yr}$ for the blue compact dwarf H1034-2558.

The integrated $\mathrm{H}_{\alpha}$ luminosity does not correlate with the total optical luminosity $L_{B}$, the relative gas content $M_{\mathrm{HI}} / L_{B}$ or even the optical colour $B-I$. These results clearly indicate that, apart from the few blue compact dwarf galaxies in the sample, the relative strength of the current star formation is very weak in the majority of the dwarfs. The stellar population is by far dominated by older stars. The presence of A-stars is most prominent in the integrated optical spectra of H1031-2818, H1033-2707 and H1034-2758, which show strong absorption Balmer lines. The equivalent width of $\mathrm{H}_{\delta}$ ranges for these galaxies between 3 and $5 \AA$ (Table 6 ). From our spectral models, 
Table 6. Integrated quantities

\begin{tabular}{cccccc}
\hline Object & $\begin{array}{c}E Q W([\mathrm{OII}] 3727) \\
\AA\end{array}$ & $\begin{array}{c}E Q W\left(\mathrm{H}_{\delta}\right) \\
\AA\end{array}$ & $\begin{array}{c}E Q W\left(\mathrm{H}_{\beta}\right) \\
\AA\end{array}$ & $\begin{array}{c}\text { flux }\left(\mathrm{H}_{\alpha}\right) \\
10^{-15} \mathrm{erg} \mathrm{cm}^{-2} \mathrm{~s}^{-1}\end{array}$ & $\begin{array}{c}L\left(\mathrm{H}_{\alpha}\right) \\
10^{39} \mathrm{erg} \mathrm{s}^{-1}\end{array}$ \\
\hline H1031-2818 & $19.5 \pm 0.6$ & $3.3 \pm 0.5$ & $5.77 \pm 0.6$ & $9.45 \pm 0.13$ & $2.51 \pm 0.03$ \\
H1031-2632 & - & - & - & $<0.26$ & $<0.07$ \\
H1032-2638 & $16.0 \pm 1.5$ & - & $3.71 \pm 1.4$ & $1.32 \pm 0.09$ & $0.36 \pm 0.02$ \\
H1032-2722 & $58.7 \pm 2.2$ & - & $12.8 \pm 1.1$ & $1.58 \pm 0.06$ & $0.42 \pm 0.02$ \\
H1033-2707 & $26.1 \pm 1.0$ & $5.1 \pm 0.5$ & $5.81 \pm 0.5$ & $3.22 \pm 0.10$ & $0.86 \pm 0.03$ \\
H1033-2642 & $21.5 \pm 1.9$ & $3.5 \pm 1.1$ & - & $0.74 \pm 0.06$ & $0.20 \pm 0.02$ \\
H1033-2722 & $38.9 \pm 2.8$ & - & - & $1.02 \pm 0.10$ & $0.27 \pm 0.03$ \\
H1034-2558 & $27.1 \pm 1.5$ & $-4.6 \pm 0.6$ & $50.0 \pm 1.8$ & $10.96 \pm 0.05$ & $3.06 \pm 0.01$ \\
H1034-2758 & $40.0 \pm 0.5$ & $4.3 \pm 0.6$ & $13.0 \pm 0.5$ & $9.13 \pm 0.11$ & $2.41 \pm 0.03$ \\
H1035-2756 & $43.3 \pm 3.3$ & $5.7 \pm 2.4$ & $8.23 \pm 2.9$ & $1.21 \pm 0.10$ & $0.32 \pm 0.03$ \\
H1035-2605 & $15.5 \pm 1.2$ & $<4.7$ & $5.20 \pm 1.1$ & $5.21 \pm 0.22$ & $1.46 \pm 0.06$ \\
H1035-2502 & $19.2 \pm 1.8$ & - & $<1.65$ & $0.65 \pm 0.08$ & $0.18 \pm 0.02$ \\
H1035-2740 & $49.4 \pm 1.5$ & $2.5 \pm 0.9$ & $21.1 \pm 1.3$ & $4.24 \pm 0.07$ & $1.12 \pm 0.02$ \\
H1038-2733 & $40.6 \pm 3.1$ & - & $9.13 \pm 2.4$ & $1.64 \pm 0.10$ & $0.43 \pm 0.03$ \\
H1038-2730 & - & - & - & $0.50 \pm 0.08$ & $0.13 \pm 0.02$ \\
\hline
\end{tabular}

Table 7. Oxygen abundances

\begin{tabular}{lccccccc}
\hline Object & $(1)$ & $(2)$ & $(3)$ & $(4)$ & $(5)$ & $(6)$ & $(7)$ \\
\hline H1031-2818 & 8.74 & - & $(7.22)$ & $(8.40)$ & 8.81 & 8.74 & 8.82 \\
H1032-2638 & 7.82 & - & 7.82 & - & $(8.21)$ & - & 8.11 \\
H1032-2722 & 7.73 & - & 7.73 & - & $(8.30)$ & - & 8.00 \\
H1033-2707 & 8.01 & - & 7.67 & 8.01 & $(8.36)$ & $(8.30)$ & 8.14 \\
H1033-2722 & 8.45 & - & $(7.51)$ & $(8.11)$ & 8.52 & 8.45 & 8.44 \\
H1034-2558 & 7.95 & 7.95 & 7.96 & 7.88 & $(8.07)$ & $(8.31)$ & - \\
H1034-2758 & 7.90 & - & 7.90 & - & $(8.13)$ & - & 8.04 \\
H1035-2756 & 7.95 & - & 7.95 & - & $(8.08)$ & - & - \\
H1035-2605 & 8.45 & - & $(7.49)$ & $(8.31)$ & 8.54 & 8.45 & 8.65 \\
H1035-2740 & 7.84 & - & 7.75 & 7.84 & $(8.28)$ & $(8.31)$ & 7.65 \\
H1038-2733 & 8.04 & - & 7.79 & 8.04 & $(8.24)$ & $(8.19)$ & - \\
\hline
\end{tabular}

(1): adopted oxygen abundance $12+\log (\mathrm{O} / \mathrm{H})(2)$ : physical abundance derived with the Te-method (3): abundance estimated from $R_{23}$ (lower branch) (4): abundance estimated with the p-method (lower branch) (5): abundance estimated from $R_{23}$ (upper branch) (6): abundance estimated with the p-method (upper branch) (7): abundance estimated from $[\mathrm{NII}] / \mathrm{H}_{\alpha}$.

we estimate the flux contribution of A stars at $4050 \AA$ to be about 10-20\%. Finally, an old extended underlying population can be seen in our $K^{\prime}$-band images (see Fig. 13 in Paper I).

\subsection{Oxygen abundance}

The oxygen abundance for a given galaxy was estimated using the oxygen emission lines of its brightest HII regions. For one galaxy, H1034-2558, the electron temperature $T e$ of the ionised gas could be properly determined and a direct physical measure of the oxygen abundance in the gas could be made. The IONIC package in IRAF was used. For the other objects, the temperature-sensitive
$[\mathrm{OIII}]_{\lambda 4363}$ line was either detected with a low signal-tonoise ratio or not at all. The abundances were thus estimated using various methods based on semi-empirical diagrams making use of the $[\mathrm{OII}]$, [OIII] and $\mathrm{H}_{\beta}$ lines. We have compared results based on the calibrations of $R 23=\left([\mathrm{OII}]_{\lambda 3727}+[\mathrm{OIII}]_{\lambda 4959}+[\mathrm{OIII}]_{\lambda 5007}\right) / \mathrm{H}_{\beta}$ by Edmunds \& Pagel (1984), updated by Pilyugin (2000) and the more recent and sophisticated ones of Pilyugin (2000) refered to as the "p-method". The latter method, which makes use of several excitation parameters, allows one to take into account the intrinsic variations of $R 23$ with the physical conditions of HII regions of the same metallicity. This problem has been emphasised, among others, by McGaugh (1991). Moreover, the semi-empirical 

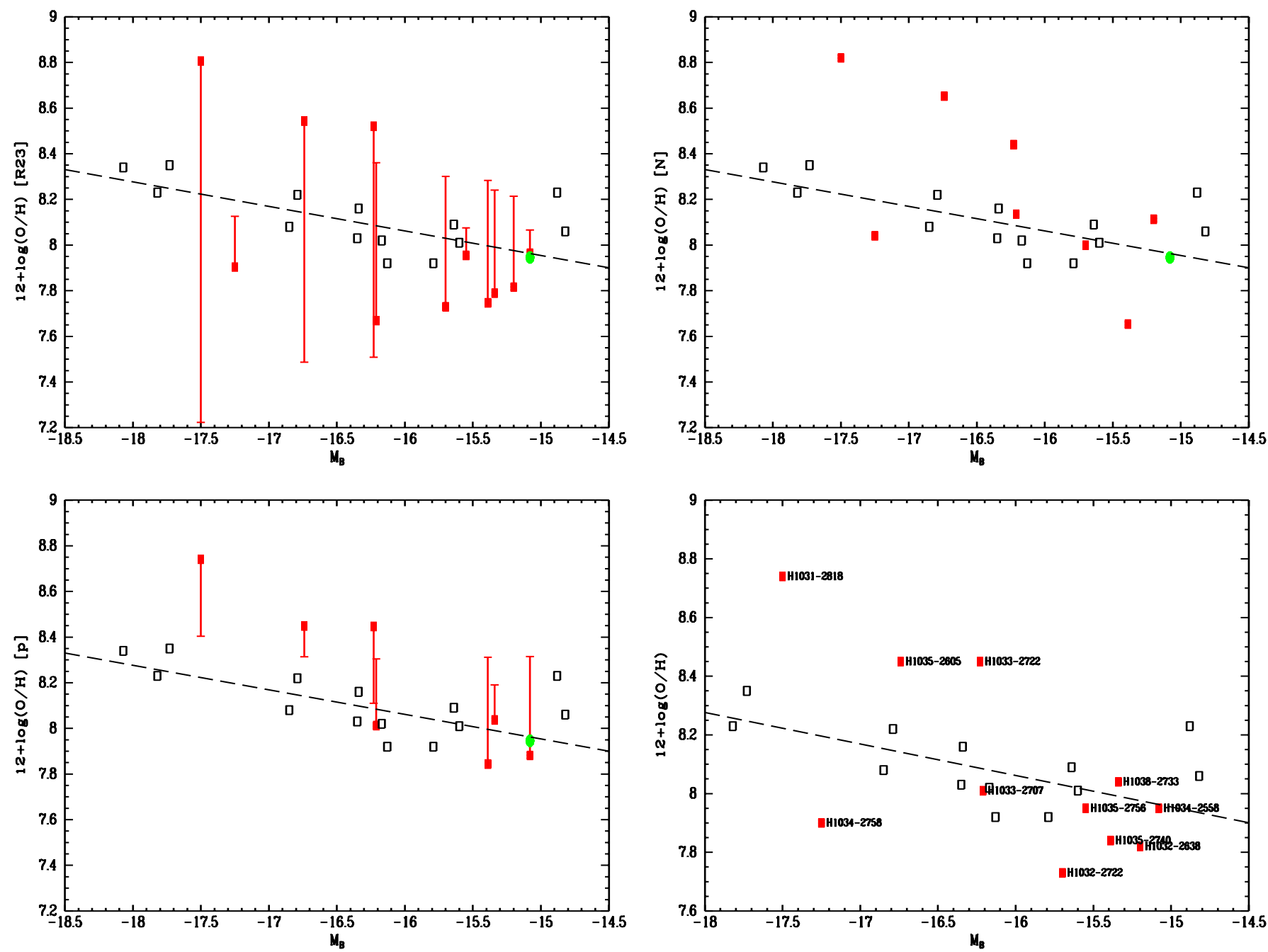

Fig. 18. Oxygen abundance vs. absolute blue magnitude of the Hydra dwarfs. Estimates using several methods are presented: semi-empirical R23 method (top-left); p-method of Pilyugin (2000) (lower-left); N/O-calibration of van Zee et al. (1998). The extremities of the bars indicate metallicities estimated using either the upper or the lower branches of the semi-empirical methods while the prefered values are shown with the filled squares. The filled circle corresponds to H1034-2558 whose oxygen abundance was determined by the physical method. Metallicities that have finally been adopted (see text) are indicated in the lower-right panel together with the galaxy identification. The sample of isolated dwarf irregular galaxies of Richer \& McCall (1995) is shown in each panel with the open squares. The dashed line is the linear fit of the corresponding metallicity-luminosity relation

techniques based on the excitation parameters suffer from a severe ambiguity: a single value of $R 23$ corresponds to two values of the oxygen abundance which is either read on the lower or upper branch of the $[\mathrm{O} / \mathrm{H}]$ vs. $R 23$ relation. To select between the two, we have used the $[\mathrm{NII}] /[\mathrm{OII}]$ line ratio as a critical diagnostic; according to McGaugh (1994), a value $\log ([\mathrm{NII}] /[\mathrm{OII}])>-1$ favours the highest metallicities. As an independent criterion, we have applied a method based on the $[\mathrm{NII}] / \mathrm{H}_{\alpha}$ line ratio, which was initially introduced by Alloin et al. (1979) and more recently calibrated by van Zee et al. (1998). In Table 7, we summarise the results obtained using the physical "Te-method" and the semi-empirical "R23" calibration (Cols. 3 and 5), "p-method" (Cols. 4 and 6) and $[\mathrm{NII}] / \mathrm{H}_{\alpha}$ calibration (Col. 7) methods. Rejected values based on the $[\mathrm{NII}] /[\mathrm{OII}]$ criterion are indicated in paren- theses. Inconsistent values given by the "p-method" have been omitted.

The errors associated with the empirical methods are at least 0.2 dex.

In general, the abundances derived from the $R 23$ and p-methods are comparable for the upper branch, whereas for the lower one they differ by up to 0.3 dex. Note that the abundance of H1034-2558, which is the only one that could be measured directly, is, within the errors, consistent with both calibrations. In principle, one may use the correlation between the metallicity and the luminosity/mass of galaxies, which is valid for both isolated dwarf and giant galaxies, to further check the accuracy of the semi-empirical techniques. Figure 18 presents plots of the oxygen abundance vs. absolute blue magnitudes for each method. The "p-method" appears to have a lower 
scatter with respect to the metallicity-luminosity relation. Moreover, it is more reliable than the classical $R 23$ one, according to Pilyugin (2000) who compared metallicities determined with both empirical and physical methods. Therefore, with the exception of H1034-2558 and whenever possible, we have adopted for further analysis the abundances estimated with this technique. They are listed in Col. 1 of Table 7 and the corresponding metallicityluminosity plot is shown in Fig. 18 (lower-right panel). Furthermore, the agreement between the abundances estimated from the $[\mathrm{OII}],[\mathrm{OIII}]$ and $[\mathrm{NII}]$ lines is rather good. On average, they differ by 0.15 dex (see Fig. 18, top-right panel).

For the three galaxies where the metallicity could be reliably derived in at least three H II regions (H1031-2818, H1035-2605 and H1035-2740) we found variations of less than 0.2 dex over the galaxy. Such a result is expected as dwarf galaxies do not usually exhibit strong metallicity gradients.

The average oxygen abundance expressed as $12+\log (\mathrm{O} / \mathrm{H})$ is $8.1\left(Z_{\odot} / 7\right)$ for our sample of $\mathrm{H}$ I-selected dwarfs in Hydra. Oxygen abundances range between 7.7 $\left(Z_{\odot} / 15 ; \mathrm{H} 1032-2722\right)$ and $8.74\left(Z_{\odot} / 1.5 ; \mathrm{H} 1031-2818\right)$, a very high value for a dwarf galaxy. Indeed, the latter galaxy is discrepant by more than 0.4 dex, i.e. significantly higher than the instrumental/method error, from the metallicity-luminosity correlation. In Paper III, we will argue that at least part of the scatter in this relation might be due to environment effects in the cluster. Note finally that, on the other hand, no extremely metal deficient galaxy (below $Z_{\odot} / 20$ ) was picked up in this blind H I survey.

\section{Conclusions}

We have presented spectrophotometric data on a complete sample of $15 \mathrm{H} \mathrm{I-selected} \mathrm{dwarf} \mathrm{galaxies} \mathrm{in} \mathrm{the} \mathrm{Hydra} \mathrm{clus-}$ ter. We found that:

(1) the optical and H I velocities are consistent. We can therefore exclude any mis-identification of the $\mathrm{HI}$ clouds detected in the original VLA survey;

(2) almost all dwarfs exhibit star-forming regions, as expected for an $\mathrm{H}$ I-selected sample;

(3) the distribution and number of $\mathrm{HII}$ regions is very diverse and not directly related to the available $\mathrm{H} \mathrm{I}$ gas content. In most cases, the brightest $\mathrm{H}$ II regions are off-center;

(4) there is a large range of $\mathrm{H} \alpha$ luminosities and hence of burst strengths;
(5) none of the Hydra dwarfs is extremely metal-deficient. A few objects have a rather high oxygen abundance for a dwarf galaxy. In a forthcoming paper, we will discuss the environmental dependence of these spectroscopic properties, also incorporating the morphological results presented in Paper I.

Acknowledgements. It is a pleasure to thank the $3.6 \mathrm{~m}$ team at la Silla who helped us with the observations, especially Pierre Leisy. The Unité Scientifique Nançay of the Observatoire de Paris is associated as USR B704 to the French Centre National de Recherche Scientifique (CNRS). Nançay also gratefully acknowledges the financial support of the European Union, the Région Centre and the Département du Cher in France. TXT acknowledges the partial financial support of NSF grant AST 96-16863, University Paris 7 and CNRS for a visiting position.

\section{References}

Alloin, D., Collin-Souffrin, S., Joly, M., \& Vigroux, L. 1979, A\&A, 78, 200

Bravo-Alfaro, H., Cayatte, V., van Gorkom, J. H., \& Balkowski, C. 2000, AJ, 119, 580

Cayatte, V., van Gorkom, J. H., Balkowski, C., \& Kotanyi, C. 1990, AJ, 100, 604

Dickey, J. M. 1997, AJ, 113, 1939

Duc, P. A., Papaderos, P., Balkowski, C., et al. 1999, A\&AS, 136, 539

Edmunds, M. G. \& Pagel, B. E. J. 1984, MNRAS, 211, 50

Filippenko, A. V. 1982, PASP, 94, 715

Fouqué, P., Durand, N., Bottinelli, L., Gouguenheim, L., \& Paturel, G. 1990, A\&AS, 86, 473

Jacoby, G. H., Hunter, D. A., \& Christian, C. A. 1984, ApJS, 56,257

Kenney, J. D. P. 1990, in The interstellar Medium in Galaxies, ed. H. A. Thronson \& M. Shull (Kluwer Academic Publishers), 151

Kennicutt, R. C., J. 1998, ARA\&A, 36, 189

Kennicutt, R. C., J. \& Hodge, P. W. 1986, ApJ, 306, 130

McGaugh, S. S. 1991, ApJ, 380, 140

McGaugh, S. S. 1994, ApJ, 426, 135

McMahon, P. M. 1993, Ph.D. Thesis, Columbia University

Pilyugin, L. S. 2000, A\&A, 362, 325

Richer, M. G. \& McCall, M. L. 1995, ApJ, 445, 642

Struck, C. 1999, Physics Reports, 321, 1

Torres-Peimbert, S., Peimbert, M., \& Fierro, J. 1989, ApJ, 345, 186

Valluri, M., van Gorkom, J., \& McMahon, P. 2001, submitted to AJ

van Zee, L., Salzer, J. J., Haynes, M. P., O’Donoghue, A. A., \& Balonek, T. J. 1998, AJ, 116, 2805

van Zee, L., Salzer, J. J., \& Skillman, E. 2001, in preparation 